Research Article

\title{
Hyperbaric Normoxia Improved Glucose Metabolism and Decreased Inflammation in Obese Diabetic Rat
}

\author{
Naoto Fujita ${ }^{(D)}{ }^{1}$ Natsuki Goto, ${ }^{1}$ Tomoya Nakamura, ${ }^{1}$ Wataru Nino, ${ }^{1}$ Taketoshi Ono, ${ }^{2}$ \\ Hisao Nishijo, ${ }^{2}$ and Susumu Urakawa $\mathbb{1}^{1}$ \\ ${ }^{1}$ Department of Musculoskeletal Functional Research and Regeneration, Graduate School of Biomedicine and Health Sciences, \\ Hiroshima University, 1-2-3 Kasumi, Minami-ku, Hiroshima 734-8553, Japan \\ ${ }^{2}$ System Emotional Science, Graduate School of Medicine and Pharmaceutical Sciences, University of Toyama, Sugitani 2630, \\ Toyama 930-0194, Japan
}

Correspondence should be addressed to Naoto Fujita; fujitan@hiroshima-u.ac.jp

Received 17 May 2019; Revised 9 October 2019; Accepted 2 November 2019; Published 19 November 2019

Academic Editor: Dario Pitocco

Copyright (C) 2019 Naoto Fujita et al. This is an open access article distributed under the Creative Commons Attribution License, which permits unrestricted use, distribution, and reproduction in any medium, provided the original work is properly cited.

Hyperbaric treatment improves hyperglycemia and hyperinsulinemia in type 2 diabetes associated with obesity. However, its mode of action is unknown. The purpose of the present study was to investigate the influences of regular hyperbaric treatment with normal air at 1.3 atmospheres absolute (ATA) on glucose tolerance in type 2 diabetes with obesity. The focus was directed on inflammatory cytokines in the adipose tissue and skeletal muscle. Otsuka Long-Evans Tokushima Fatty (OLETF) rats were used as models of type 2 diabetes with obesity and Long-Evans Tokushima Otsuka (LETO) rats served as healthy controls. The rats were randomly assigned to untreated or hyperbaric treatment groups exposed to 1.3 ATA for $8 \mathrm{~h} \mathrm{~d}^{-1}$ and $5 \mathrm{~d}^{-1}$ for 16 wks. Glucose levels were significantly higher in the diabetic than in the healthy control rats. Nevertheless, glucose levels at 30 and 60 min after glucose administration were significantly lower in the diabetic rats treated with 1.3 ATA than in the untreated diabetic rats. Insulin levels at fasting and $120 \mathrm{~min}$ after glucose administration were significantly lower in the diabetic rats treated with 1.3 ATA than in the untreated diabetic rats. Hyperbaric treatment also increased interleukin-10 (IL-10) expression in the skeletal muscle and decreased tumor necrosis factor $\alpha(\mathrm{TNF} \alpha)$ expression in adipose tissue. These results suggested that $\mathrm{TNF} \alpha$ downregulation and IL-10 upregulation in diabetic rats subjected to hyperbaric treatment participate in the crosstalk between the adipose and skeletal muscle tissues and improve glucose intolerance.

\section{Introduction}

Nutrient overload induces low-grade chronic inflammation of the adipose tissue, skeletal muscle, liver, pancreas, and hypothalamus. Insulin resistance and glucose intolerance develop as a consequence of this inflammation [1]. Insulin receptors and inflammatory signaling interact at the insulin receptor substrate level. Proinflammatory cytokines released from adipose and other inflammatory tissues interfere with glucose uptake via the insulin signaling pathway [2]. It is now recognized that inflammation associated with chronic obesity leads to type 2 diabetes [3].

Medication [4], diet [5], and exercise [6] are the key therapeutic approaches to the management of type 2 diabetes with obesity. They improve insulin resistance and glucose intolerance and reduce inflammation. Previous studies have demonstrated the efficacy of anti-inflammatory therapy and other strategies targeting proinflammatory cytokines such as interleukin-6 (IL-6) [7], tumor necrosis factor $\alpha$ (TNF $\alpha$ ) [8], and interleukin-1 $\beta$ (IL-1 $\beta$ ) [9]. Medication therapy is a critical component of the treatment of patients with obese diabetes. However, this approach is costly. Therefore, the application of a low-cost complementary treatment such as physical exercise is recommended for the clinical management of this disorder.

The benefits of regular physical exercise for the treatment of patients with type 2 diabetes and obesity are widely known. The American College of Sports Medicine recommends regular aerobic exercise and resistance training to improve insulin resistance and glucose intolerance in diabetic patients 
[10]. We confirmed the efficacy of physical exercise in a rat model of type 2 diabetes with obesity [11]. As many patients dislike physical activity, however, this therapeutic approach is often inconsistently practiced.

It was reported that hyperbaric treatment stimulates the skeletal muscle and improves insulin resistance and glucose intolerance. It maintains elevated atmospheric pressure and oxygen concentration with an air compressor and an oxygen concentrator. It enhances the partial pressure of oxygen and increases the concentration of dissolved oxygen in the blood [12]. We confirmed the efficacy of hyperbaric treatment in type 2 diabetes. This therapy increases oxidative metabolism in the skeletal muscles [13-15]. Hyperbaric treatment requires no physical activity on the part of patients with type 2 diabetes and obesity. Therefore, they may be more likely to submit to it. Recently, hyperbaric treatment with normal air at $<2$ atmospheres absolute (ATA) has been substituted for concentrated oxygen and is gaining popularity as a home remedy. However, it remains to be determined whether hyperbaric treatment using normal air at $<2$ ATA is as effective at improving obese type 2 diabetes as concentrated oxygen. Moreover, the effects of hyperbaric treatment on inflammatory cytokines in type 2 diabetes with obesity remain unknown. Hyperbaric treatment using normal air is usually performed at 1.3 ATA, and this condition is now very common for the convenience of users. The purpose of the present study was to investigate the influences of regular hyperbaric treatment using normal air at 1.3 ATA on glucose tolerance in type 2 diabetes with obesity. This research also focused on inflammatory cytokines in the adipose tissue and skeletal muscle.

\section{Materials and Methods}

2.1. Experimental Design. This study was approved by the Institutional Animal Care and Use Committee of Hiroshima University (A16-5) and conducted in accordance with the Hiroshima University Regulations for Animal Experimentation. All experiments complied with the National Institute of Health Guidelines for the Care and Use of Laboratory Animals.

Male Otsuka Long-Evans Tokushima Fatty (OLETF) rats $(n=12)$ were used as models of type 2 diabetes with obesity. Diabetic rats aged $24 \mathrm{wks}$ were randomly assigned either to an untreated (Diabetes, $n=6$ ) or a hyperbaric treatment (Diabetes HB, $n=6$ ) group. The Diabetes group was further divided into subgroups according to the body weights (Diabetes obesity, $n=4$; Diabetes lean, $n=2$ ). In the Diabetes obesity group, the body weights were higher at the end of the experiment than at the start of the experiment. Conversely, in the Diabetes lean group, the body weights were lower at the end of the experiment than at the start of the experiment. Age-matched male Long-Evans Tokushima Otsuka (LETO) rats $(n=12)$ were used as healthy controls, and the rats were also randomly assigned either to an untreated (Control, $n=6$ ) or a hyperbaric treatment (Control $\mathrm{HB}, n=6)$ group. The rats were housed in a controlled environment with a fixed $12 \mathrm{~h}$ light-dark cycle (lights on from 08:00 to 20:00) and a constant temperature of $22 \pm 2$ ${ }^{\circ} \mathrm{C}$. They were fed a standard diet composed of $79 \mathrm{~g} \mathrm{~kg}^{-1}$ mois- ture, $231 \mathrm{~g} \mathrm{~kg}^{-1}$ protein, $51 \mathrm{~g} \mathrm{~kg}^{-1}$ fat, $58 \mathrm{~g} \mathrm{~kg}^{-1}$ ash, $28 \mathrm{~g} \mathrm{~kg}^{-1}$ fiber, and $553 \mathrm{~g} \mathrm{~kg}^{-1}$ carbohydrates. Food and water were provided ad libitum.

2.2. Hyperbaric Treatment. From ages $24-40$ wks, the rats in the Diabetes $\mathrm{HB}$ and Control $\mathrm{HB}$ groups were exposed to 1.3 ATA (1317.225 hPa) for $8 \mathrm{~h} \mathrm{~d}^{-1}$ (23:30-07:30), $5 \mathrm{~d} \mathrm{wk}^{-1}$. Hyperbaric treatment at 1.3 ATA is one of the complementary treatments at home. We are assuming the treatment will be used during sleep. Therefore, treatment time was determined for $8 \mathrm{~h}$ a day. The cages of the nontreated Diabetes and Control groups were placed in a hyperbaric chamber set to normal atmospheric pressure $(1013.25 \mathrm{hPa})$ to equalize compressor noise. The food intake levels of both the nontreated and treated groups were equal.

2.3. Oral Glucose Tolerance Test. At $48 \mathrm{~h}$ after the final hyperbaric treatment, an oral glucose tolerance test (OGTT) was performed to determine glucose tolerance relative to body weight. The rats were fasted for $12 \mathrm{~h}$, and glucose $\left(2 \mathrm{~g} \mathrm{~kg}^{-1}\right.$ body weight) was administered via an esophageal feeding tube. Blood samples were obtained from the lateral caudal vein before and at 30, 60, and 120 min after glucose administration. The blood samples were centrifuged at $3000 \mathrm{rpm}$ for $10 \mathrm{~min}$ at room temperature, and the plasma fraction was stored at $-80^{\circ} \mathrm{C}$ until subsequent analysis. Glucose and insulin concentrations were measured with commercially available spectrophotometric (298-65701; Wako, Osaka, Japan) and enzyme-linked immunosorbent (ELISA) (M1101; Morinaga, Yokohama, Japan) assay kits, respectively, according to the manufacturers' instructions.

As a supplementary experiment, male Sprague-Dawley rats aged $20-21 \mathrm{wks}(n=6)$ were used to determine the immediate effects of the hyperbaric treatment. After their fasting blood glucose levels were measured, the rats were exposed to 1.3 ATA for $60 \mathrm{~min}$ and their blood glucose levels were measured again.

2.4. Tissue Sampling. At $48 \mathrm{~h}$ after the OGTT, the rats were fasted for $12 \mathrm{~h}$ and sacrificed by sodium pentobarbital overdose. The epididymal adipose tissue, plantaris muscle, and soleus muscle were removed, weighed, frozen in liquid nitrogen, and stored at $-80^{\circ} \mathrm{C}$ until further analysis. The tissue samples were used to study mRNA expressions and to make frozen sections for the skeletal muscles.

2.5. Blood Analysis. Plasma samples collected at fasting were used to determine triacylglycerol (TAG) and free fatty acid (FFA) concentrations. TAG and FFA were measured with commercially available spectrophotometric assay kits (29463601 and 290-63701, respectively; Wako, Osaka, Japan) according to the manufacturer's instructions.

2.6. Histological Analysis. Portions of the epididymal adipose tissue were removed, fixed with $4 \%$ paraformaldehyde in $0.1 \mathrm{M}$ phosphate buffer, and embedded in paraffin. Tissue sections were stained with hematoxylin and eosin. The diameters of $\geq 100$ adipose cells/rat in two randomly chosen fields were measured with Image (NIH, Bethesda, MD, USA). 
Transverse sections of the middle part of the muscle belly in the plantaris and soleus muscles were obtained with a cryostat. The sections were stained for myofibrillar adenosine triphosphatase (ATPase, $\mathrm{pH}$ 4.55) activity to categorize the muscle fiber as type I, IIA, or IIB based on a previous study [16]. The cross-sectional areas of the dominant muscle fiber (type IIB and type I fibers in the plantaris and soleus muscles, respectively) were measured for three randomly chosen fields with ImageJ (NIH, Bethesda, MD, USA). For each rat, $\geq 100$ muscle fibers were measured.

\subsection{Quantitative Polymerase Chain Reaction (qPCR)} Analysis. The tissue samples to study mRNA expressions were extracted at $48 \mathrm{~h}$ after OGTT. Total RNA was isolated with a TRIzol reagent (15596-026; Invitrogen, Tokyo, Japan). The total RNA concentration was determined with a spectrophotometer (NanoDrop; Thermo Fisher Scientific, Waltham, MA, USA) and calibrated to be equal for all samples. Reverse transcription was performed with a High-Capacity cDNA Reverse Transcription Kit (4374966; Applied Biosystems, Foster City, CA, USA). The cDNA samples were stored at $-20^{\circ} \mathrm{C}$ until subsequent analysis.

The mRNA expression levels of IL-6 (Rn01410330_m1), TNF $\alpha$ (Rn01525859_g1), IL-1 $\beta$ (Rn00580432_m1), and IL10 (Rn00563409_m1) were quantified by qPCR with TaqMan Gene Expression Assays (Applied Biosystems, Foster City, CA, USA). Relative expression levels were inferred by normalizing the quantity of the cDNA template per gene against the quantity of cDNA for the 18S (Rn03928990_g1) reference standard. The cDNA concentration at each $\mathrm{qPCR}$ cycle was plotted to obtain a standard curve. The relative gene expression corresponded to the value at the threshold line. The qPCR was performed with a PCR Fast Advanced Master Mix (Applied Biosystems, Foster City, CA, USA) in CFX96 (Bio-Rad Laboratories, Hercules, CA, USA).

2.8. Near-Infrared Spectroscopy (NIRS) Analysis of Skeletal Muscle Hemodynamic Response to Hyperbaric Treatment. Male OLETF $(649 \pm 18 \mathrm{~g}, n=3)$ and LETO $(546 \pm 0 \mathrm{~g}$, $n=3$ ) rats aged 36 wks were used to assess skeletal muscle hemodynamics during hyperbaric treatment. The rats were lightly anesthetized by intraperitoneal sodium pentobarbital administration $\left(25 \mathrm{mg} \mathrm{kg}^{-1}\right)$. The NIRS probe was placed on the muscle belly in the gastrocnemius lateralis muscle and secured with adhesive tape. The rats were restrained with a plastic holder. Measurements began $40 \mathrm{~min}$ after light anesthesia induction. The concentrations of oxyhemoglobin $(\mathrm{Oxy}-\mathrm{Hb})$ and deoxyhemoglobin (Deoxy-Hb) were measured in the calf muscles $3-5 \mathrm{~mm}$ beneath the probe. Total hemoglobin (Total-Hb: sum of Oxy-Hb and Deoxy-Hb) and oxygen saturation $\left(\mathrm{StO}_{2}\right.$ : percentage of $\mathrm{Oxy}-\mathrm{Hb}$ in Total- $\mathrm{Hb}$ ) were calculated from these concentrations. Measurements were performed at normal atmospheric pressure (1 ATA) for $5 \mathrm{~min}$, then at 1.3 ATA for $10 \mathrm{~min}$. Hemodynamics was analyzed with BOM-L1TRSF (Omegawave, Tokyo, Japan).

2.9. Statistical Analysis. Data are means \pm SEM. Significant differences between group means were identified by two- way ANOVA. In a supplementary experiment, the significant differences between nontreatment and the hyperbaric treatment were evaluated using an independent $t$-test. Statistical significance was set at $p<0.05$. All statistical analyses were performed using SPSS statistical analysis software (IBM SPSS Statistics version 19.0; IBM Japan, Tokyo, Japan).

\section{Results}

3.1. Glucose and Insulin Levels during OGTT. Glucose levels were significantly higher in the diabetic rats than in the healthy control rats at fasting, 30,60, and $120 \mathrm{~min}$ after glucose administration (Figure 1(a)). However, the glucose levels at 30 and $60 \mathrm{~min}$ after glucose administration were significantly lower in the Diabetes HB group than in the Diabetes group. Insulin levels were significantly higher in the diabetic rats than in the healthy control rats at fasting, 30, 60 , and $120 \mathrm{~min}$ after glucose administration (Figure 1(b)). No significant differences in insulin level were noted between the Diabetes and Diabetes HB groups.

Throughout the experimental period, body weight was significantly higher for the diabetic rats than for the healthy control rats (Figure 1(c)). Body weight at the end of the experiment was slightly higher for the Diabetes HB group than for the Diabetes group (Diabetes, $663 \pm 99$ g; Diabetes $\mathrm{HB}, 697 \pm 38 \mathrm{~g}$ ). Although the body weight increased daily for four rats (Diabetes obesity subgroup) in the Diabetes group, the body weights of two rats (Diabetes lean subgroup) in the Diabetes group gradually decreased after the middle of the experimental period (Figure 1(d)). The mean body weights at the end of the experiment were $729 \pm 23 \mathrm{~g}$ and $530 \pm 42 \mathrm{~g}$ in the obese and lean subgroups of the Diabetes group, respectively. The body weight increased daily for all rats in the Diabetes HB group. The glucose levels were always higher in the Diabetes lean than in the Diabetes obese subgroup (Figure 1(e)). Conversely, the insulin levels were always lower in the Diabetes lean than in the Diabetes obese subgroup (Figure 1(f)). The insulin levels at fasting and 120 min after glucose administration were significantly lower in the Diabetes HB group than in the obesity subgroup of the Diabetes group.

Fasting plasma TAG (Figure $1(\mathrm{~g})$ ) and FFA (Figure 1(h)) levels were significantly higher in the diabetic than in the healthy control rats. No significant differences in the TAG and FFA levels were noted between the Diabetes and Diabetes $\mathrm{HB}$ groups.

3.2. Glucose Levels Immediately after Hyperbaric Treatment. A supplementary experiment revealed that neither nontreatment nor the hyperbaric treatment decreased the glucose levels immediately following glucose administration. In the absence of hyperbaric treatment, the mean glucose levels at fasting and at $60 \mathrm{~min}$ after glucose administration were $110 \pm 9$ $\mathrm{mgdL}^{-1}$ and $154 \pm 11 \mathrm{mgdL}^{-1}$, respectively. With hyperbaric treatment, the mean glucose levels at fasting and at $60 \mathrm{~min}$ after glucose administration were $116 \pm 6 \mathrm{mg}^{-1}$ and $156 \pm 13 \mathrm{mg}^{-1}$, respectively. There was no significant difference between the rats with and without hyperbaric treatment in terms of glucose level at $60 \mathrm{~min}$ after glucose administration. 


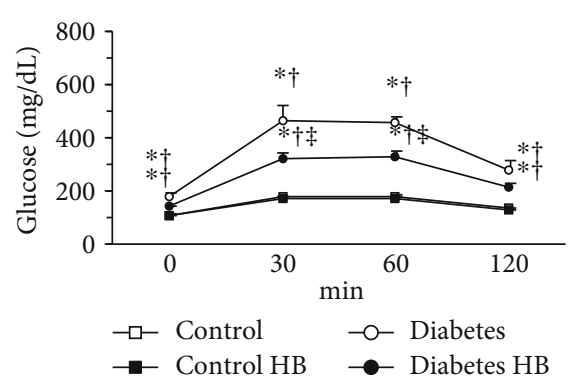

(a)

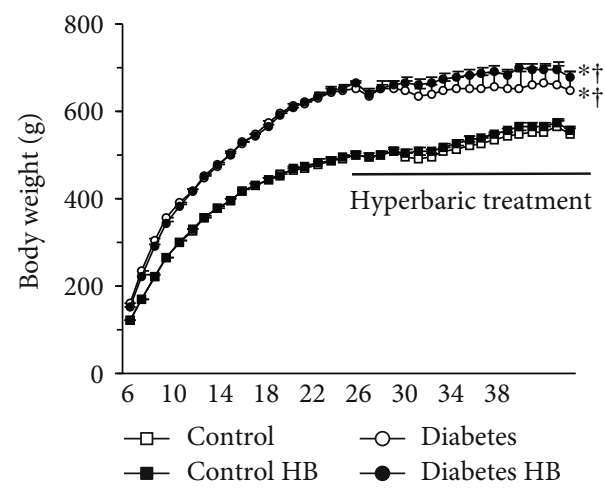

(c)

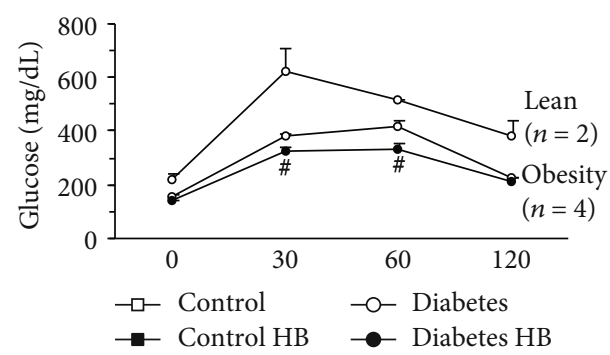

(e)

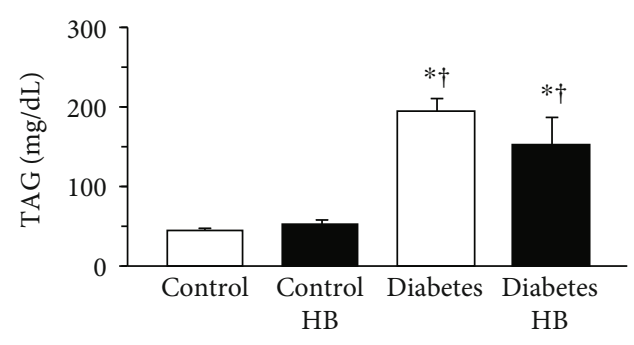

(g)

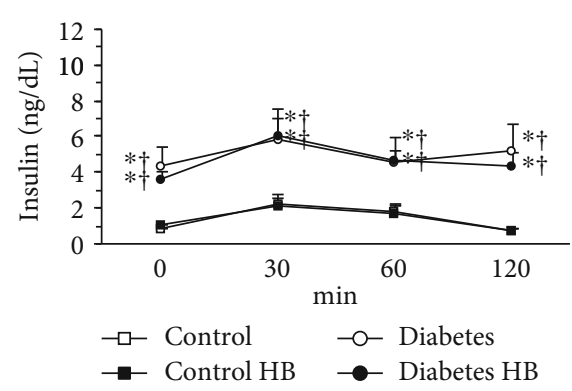

(b)

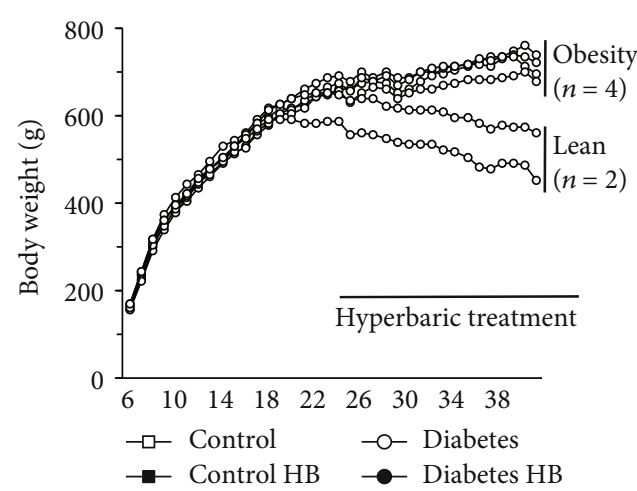

(d)

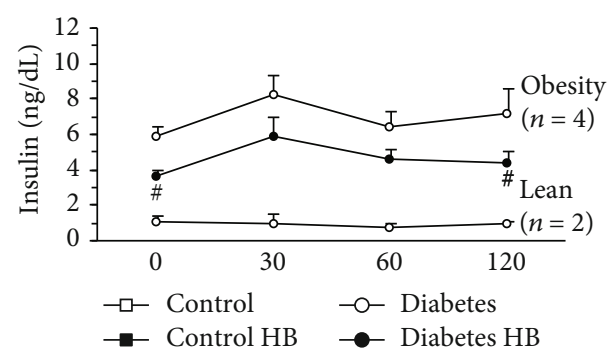

(f)

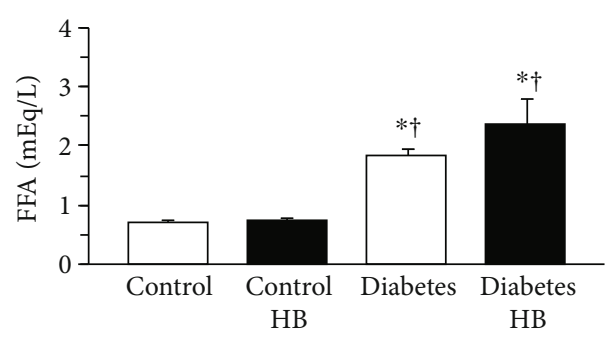

(h)

FIGURE 1: Glucose tolerance, insulin sensitivity, and metabolic properties. Glucose levels (a) and insulin levels (b) during OGTT, time course of body weight changes (c), values for the diabetes group (d-f), fasting plasma TAG levels ( $\mathrm{g}$ ), and FFA levels (h). Control: nontreated control group; Control HB: control treated with the hyperbaric normoxia group; Diabetes: nontreated diabetes group; Diabetes HB: diabetes treated with the hyperbaric normoxia group. The Diabetes group was further divided into the Diabetes obesity and Diabetes lean subgroups. Time zero: fasting. Values are means \pm standard deviation. ${ }^{*,+,}$ Significantly different from the Control, Control HB, and

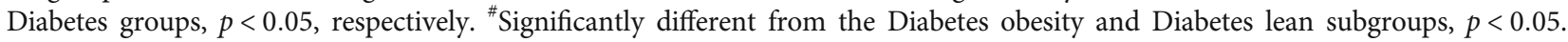

3.3. Epididymal Adipose Tissue. Adipocyte enlargement was more frequently observed in the diabetic than in the healthy control rats. However, hypertrophic adipocytes $>$ $130 \mu \mathrm{m}$ in diameter were rare in both cases (Figure 2(a)). Adipocyte diameters were significantly larger in the diabetic than in the healthy control rats (Figure $2(\mathrm{~b})$ ). The mean adipocyte diameter in the Diabetes group was $1.16 \mathrm{x}$ greater than that in the Control group. There was no significant difference between the Diabetes and Diabetes HB groups in terms of adipocyte diameter. The wet weight of epididymal adipose tissue was significantly greater in the diabetic than in the healthy control rats (Figure $2(\mathrm{c})$ ). The mean epididymal 

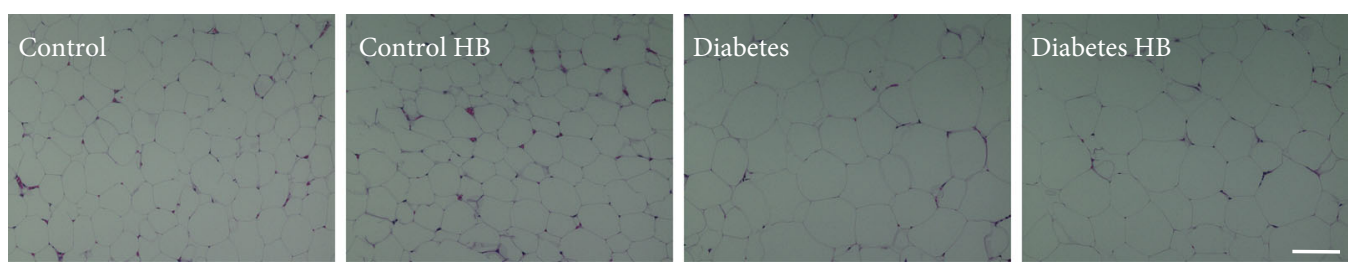

(a)

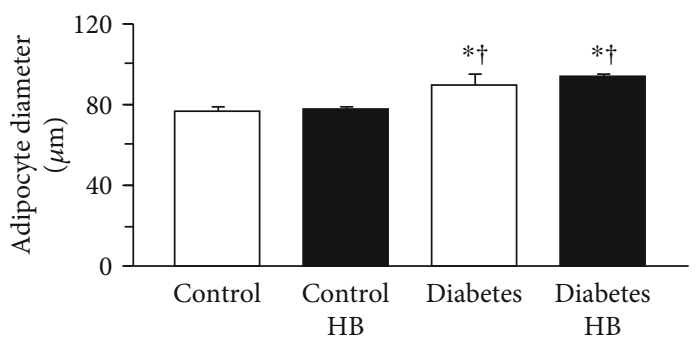

(b)

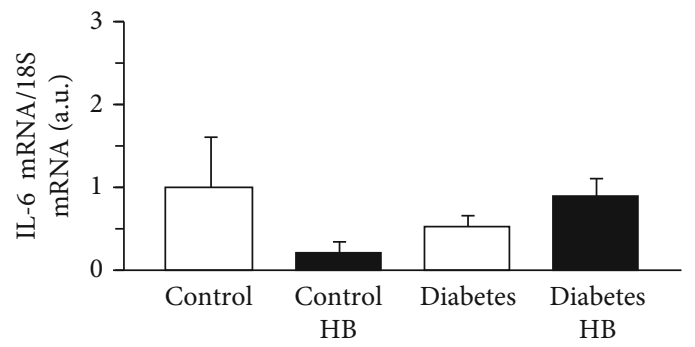

(d)

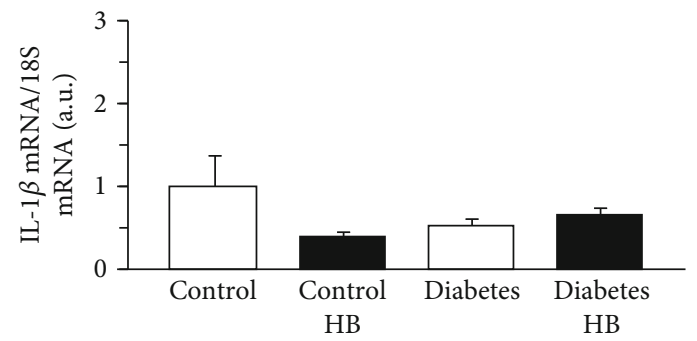

(f)

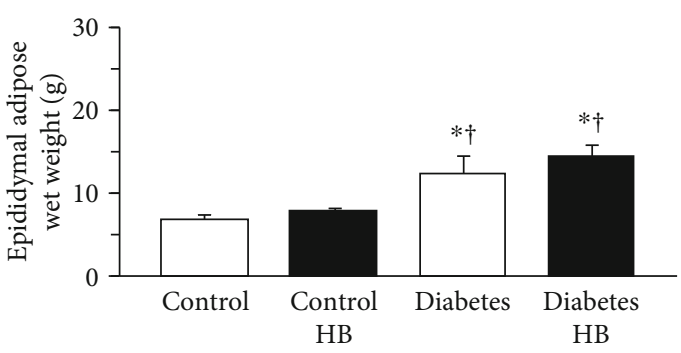

(c)

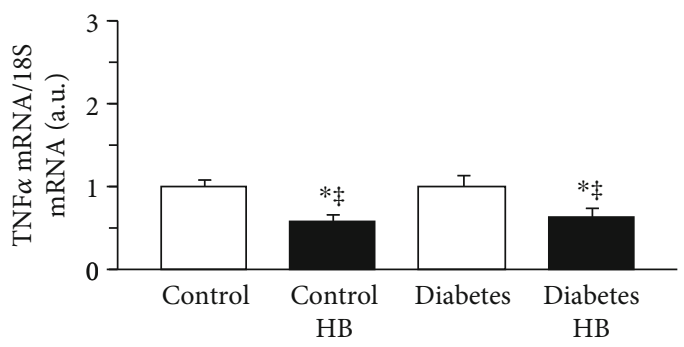

(e)

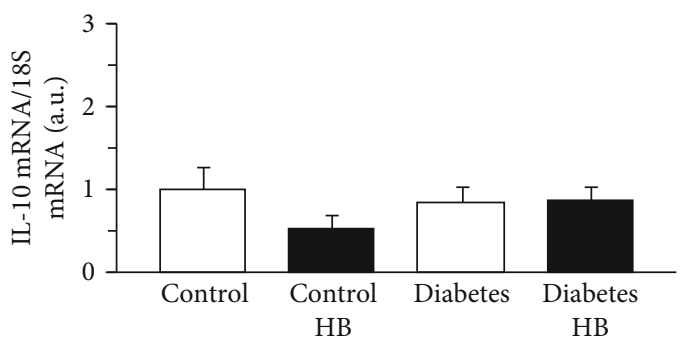

(g)

Figure 2: Adipocyte size, adipose tissue mass, and cytokine expression in adipose tissue. Representative sections of epididymal adipose tissue stained with hematoxylin and eosin (a), adipocyte diameter (b), wet weight of adipose tissue (c), expression levels of IL-6 mRNA (d), TNF $\alpha$ mRNA (e), IL-1 $\beta$ mRNA (f), and IL-10 mRNA (g). Control: nontreated control group; Control HB: control treated with the hyperbaric normoxia group; Diabetes: nontreated diabetes group; Diabetes HB: diabetes treated with the hyperbaric normoxia group. Bar $=100 \mu \mathrm{m}$. Values are means \pm standard deviation. IL-6, TNF $\alpha$, IL- $1 \beta$, and IL-10 mRNA expression levels were calculated as fold changes relative to the Control group. ${ }^{*, \dagger, \ddagger}$ Significantly different from the Control, Control HB, and Diabetes groups, $p<0.05$, respectively.

adipose tissue weight in the Diabetes group was $1.78 \mathrm{x}$ larger than that in the Control group. There was no significant difference between the Diabetes and Diabetes HB groups in terms of the wet weight of epididymal adipose tissue.

The expression levels of IL- $6, \mathrm{TNF} \alpha$, IL- $1 \beta$, and IL-10 mRNA are shown in Figures $2(\mathrm{~d})-2(\mathrm{~g})$, respectively. There were no significant differences among groups in terms of the expression levels of IL-6, IL- $1 \beta$, and IL-10 mRNA. On the other hand, the expression levels of TNF $\alpha$ were significantly lower in the rats exposed to the hyperbaric environment than in those not exposed to it (Figure 2(e)).
3.4. Skeletal Muscle. ATPase staining revealed that the plantaris muscles were composed of type I, IIA, and IIB fibers (Figure 3(a)). Type IIB fiber predominated in all groups. The cross-sectional area of the type IIB fiber in the diabetic rats was significantly smaller than that in the healthy control rats (Figure 3(b)). There was no significant difference between the Diabetes and Diabetes $\mathrm{HB}$ groups in terms of the cross-sectional area of the type IIB fiber. The wet weight of the plantaris muscle was significantly lower in the diabetic than in the healthy control rats (Figure 3(c)). There was no significant difference between 

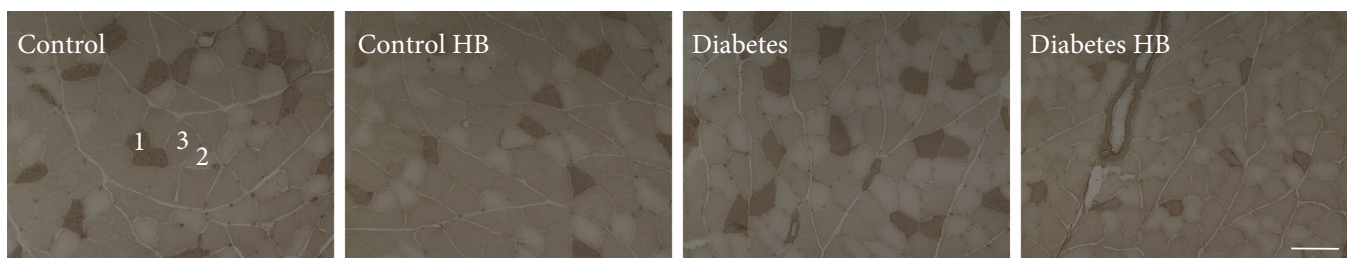

(a)

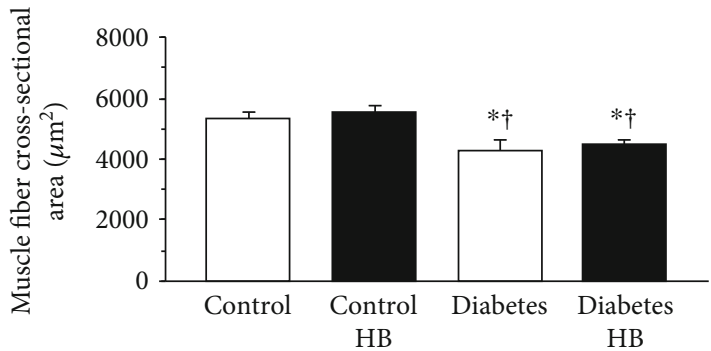

(b)

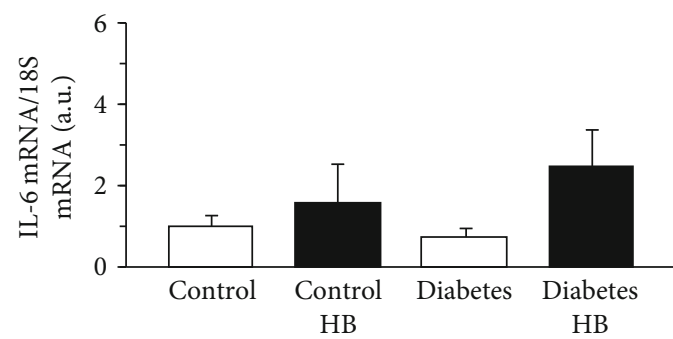

(d)

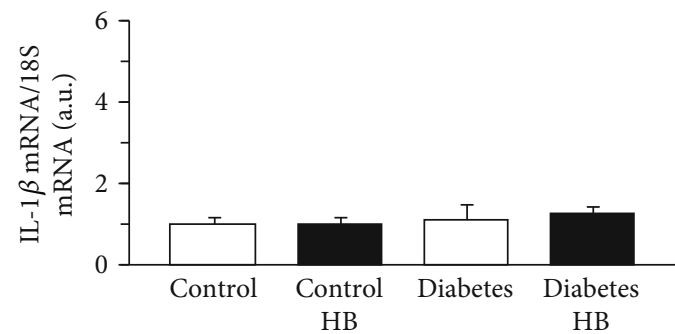

(f)

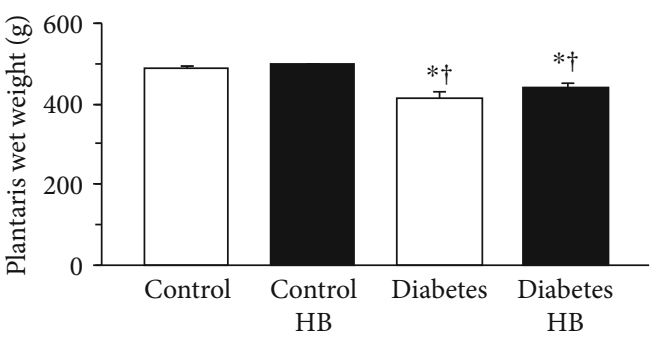

(c)

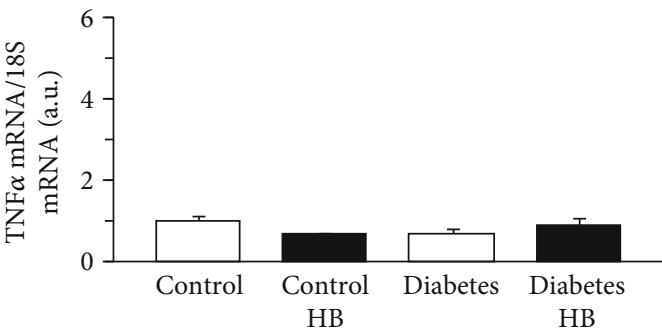

(e)

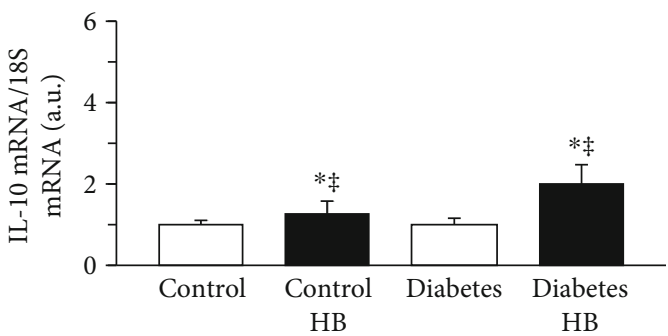

(g)

Figure 3: Muscle fiber cross-sectional area, wet weight, and cytokine expression in the plantaris muscle. Representative sections of the plantaris muscle stained for ATPase activity (a), type IIB fiber cross-sectional area (b), wet weight (c), expression levels of IL-6 mRNA (d), TNF $\alpha$ mRNA (e), IL-1 $\beta$ mRNA (f), and IL-10 mRNA (g). Control: nontreated control group; Control HB: control treated with the hyperbaric normoxia group; Diabetes: nontreated diabetes group; Diabetes HB: diabetes treated with the hyperbaric normoxia group; 1: type I fiber; 2: type IIA fiber; 3: type IIB fiber. Bar $=100 \mu \mathrm{m}$. Values are means \pm standard deviation. IL-6, TNF $\alpha$, IL- $1 \beta$, and IL-10 mRNA expression levels were calculated as fold changes relative to the Control group. ${ }^{*, \dagger, \ddagger}$ Significantly different from the Control, Control HB, and Diabetes groups, $p<0.05$, respectively.

the Diabetes and Diabetes HB groups in terms of the wet weight of the plantaris muscle.

The expression levels of IL-6, TNF $\alpha$, IL-1 $\beta$, and IL-10 mRNA in the plantaris muscle are shown in Figures 3(d)$3(\mathrm{~g})$, respectively. There were no significant differences among groups in terms of the expression levels of IL-6, TNF $\alpha$, and IL- $1 \beta$ mRNA. Nevertheless, the expression levels of IL-10 were significantly higher in rats exposed to the hyperbaric environment than in those not exposed to it (Figure 3(g)).

The soleus muscles were composed of type I and IIA fibers (Figure 4(a)) with the type I fiber predominating in all groups. The muscle fiber cross-sectional area and the wet weight of the soleus muscle resembled those of the plantaris muscle. The cross-sectional area of the type I fiber (Figure 4(b)) and the wet weight of the soleus muscle (Figure 4(c)) were significantly smaller in the diabetic than in the healthy control rats. There were no significant differences between the Diabetes and Diabetes HB groups in terms of the cross-sectional area of the type I fiber and the wet weight of the soleus muscle.

The expression levels of IL- 6 , TNF $\alpha$, IL- $1 \beta$, and IL-10 mRNA in the soleus muscle are shown in Figures 4(d)-4(g), respectively. There were no significant differences among groups in terms of the expression levels of IL- 6, TNF $\alpha$, and 

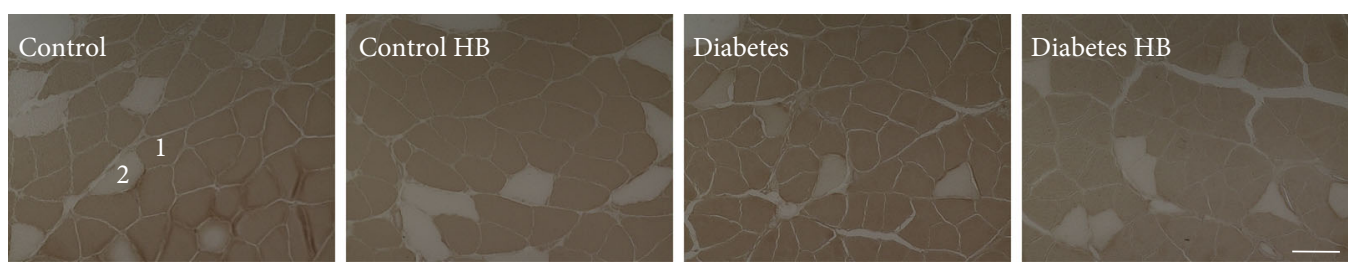

(a)

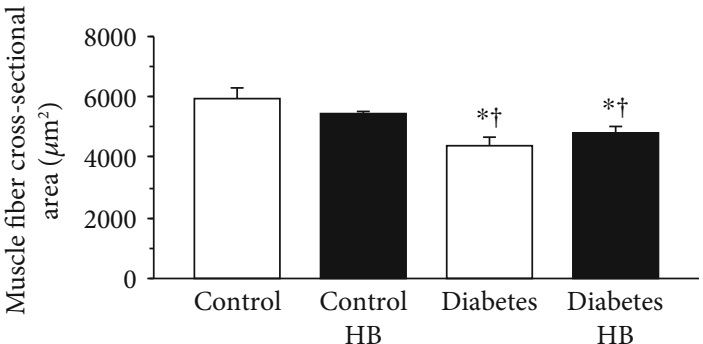

(b)

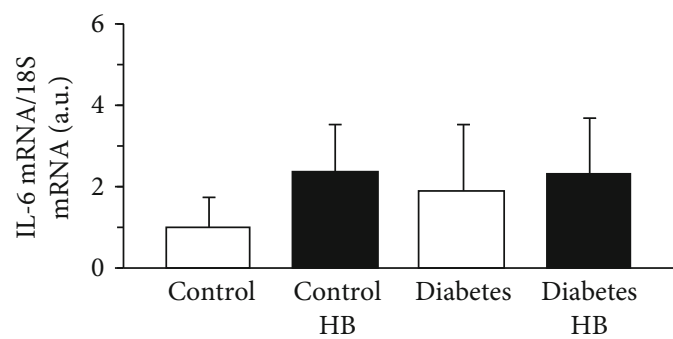

(d)

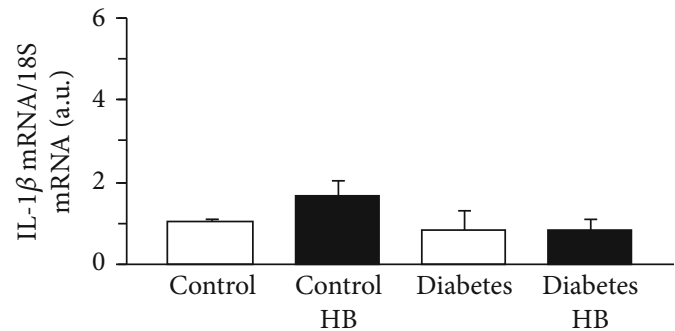

(f)

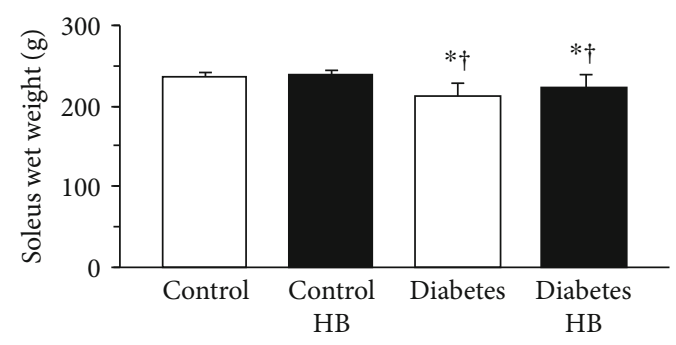

(c)

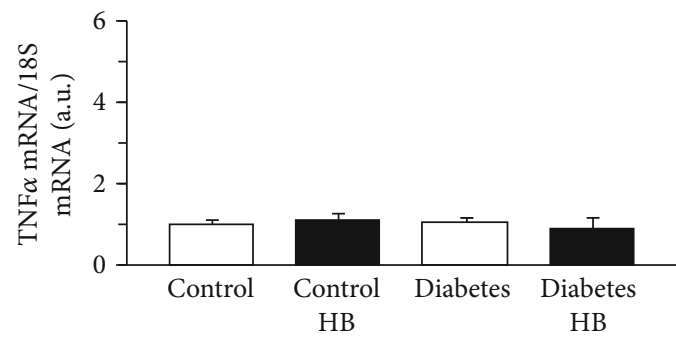

(e)

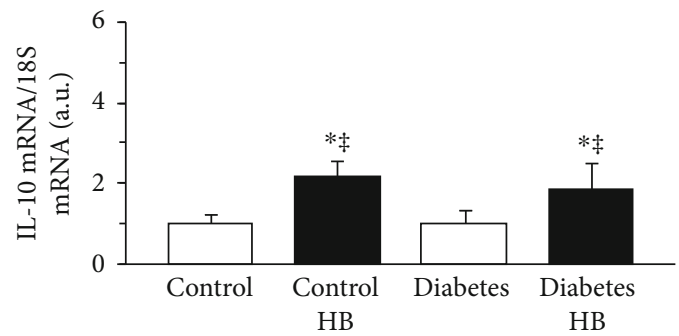

(g)

FIGURE 4: Muscle fiber cross-sectional area, wet weight, and cytokine expression in the soleus muscle. Representative sections of the soleus muscle stained for ATPase activity (a), type I fiber cross-sectional area (b), wet weight (c), expression levels of IL-6 mRNA (d), TNF $\alpha$ mRNA (e), IL-1 $\beta$ mRNA (f), and IL-10 mRNA (g). Control: nontreated control group; Control HB: control treated with the hyperbaric normoxia group; Diabetes: nontreated diabetes group; Diabetes HB: diabetes treated with the hyperbaric normoxia group; 1: type I fiber; 2: type IIA fiber. Bar $=100 \mu \mathrm{m}$. Values are means \pm standard deviation. IL-6, TNF $\alpha$, IL- $1 \beta$, and IL- 10 mRNA expression levels were calculated as fold changes relative to the Control group. ${ }^{*, \dagger, \ddagger}$ Significantly different from the Control, Control HB, and Diabetes groups, $p<0.05$, respectively.

IL-1 $\beta$ mRNA. However, the expression levels of IL-10 were significantly higher in rats exposed to the hyperbaric environment than in those not exposed to it (Figure 4(g)).

\subsection{Skeletal Muscle Hemodynamic Response to Hyperbaric} Treatment. Hyperbaric treatment substantially elevated Oxy$\mathrm{Hb}$, slightly reduced Deoxy- $\mathrm{Hb}$, and increased $\mathrm{StO}_{2}$ in the calf muscles (Figure 5). The mean $\mathrm{Oxy}-\mathrm{Hb}$ was significantly higher at 1.3 ATA than at 1.0 ATA (Figure 6(a)). There were no significant differences between the 1.0 ATA and 1.3 ATA treatments in terms of the mean Deoxy-Hb (Figure 6(b)) or mean Total-
$\mathrm{Hb}$ (Figure 6(c)). The mean $\mathrm{StO}_{2}$ was significantly higher at 1.3 ATA than at 1.0 ATA (Figure 6(d)).

\section{Discussion}

In the present study, regular hyperbaric normoxia at 1.3 ATA improved glucose levels at 30 and $60 \mathrm{~min}$ after glucose administration and insulin levels at fasting and $120 \mathrm{~min}$ after glucose administration in a rat model of type 2 diabetes with obesity. It also upregulated IL-10 in the skeletal muscle and downregulated TNF $\alpha$ in adipose tissue. 


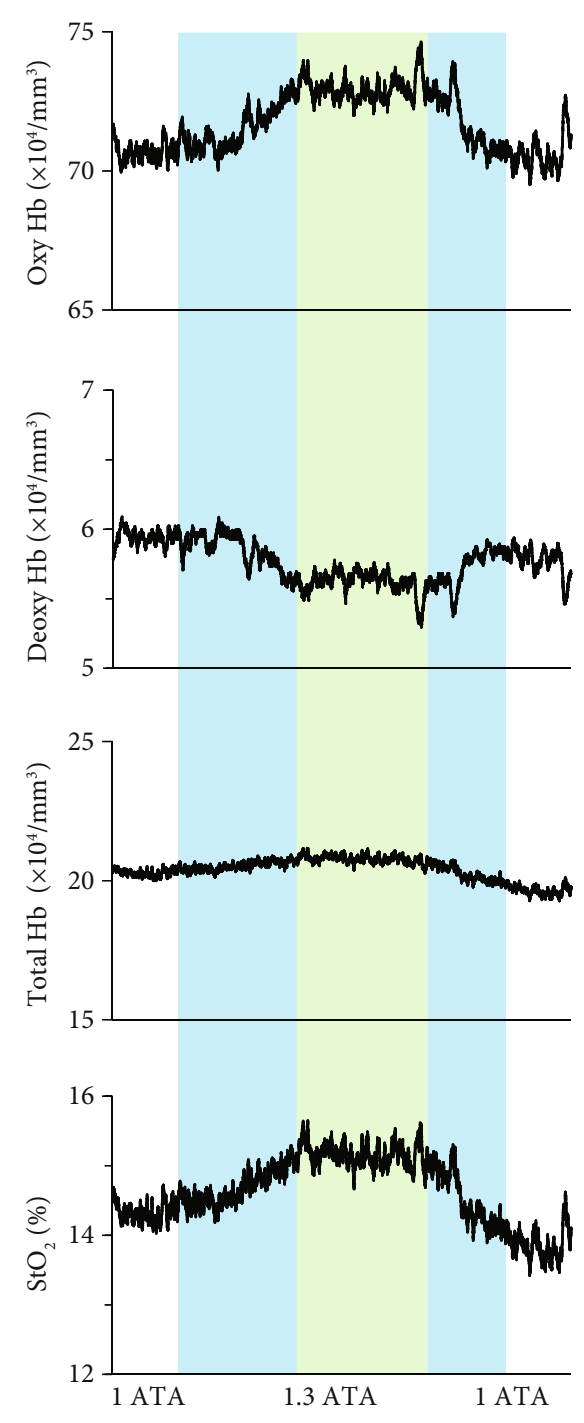

FIGURE 5: Representative NIRS data for calf muscles during hyperbaric treatment. The green area indicates 1.3 ATA, and blue areas indicate early compression and late decompression periods. Each variable was sequentially calculated every $0.1 \mathrm{~s}$.

OLETF rats are missing the satiety signal receptor in the ventromedial nucleus of the hypothalamus. Consequently, they develop hyperphagia and eventually become obese [17]. Obesity is associated with increased expression of proinflammatory cytokines such as IL-6, TNF $\alpha$, and IL$1 \beta$ in adipose tissue. These factors have detrimental effects on the insulin signaling pathway [18]. Obesity-related insulin resistance and glucose intolerance have been attributed to defects in the insulin signaling pathway [19]. In the present study, there were no significant differences between the diabetic and healthy control rats in terms of the expression levels of IL- 6 , TNF $\alpha$, and IL- $1 \beta$ mRNA per unit adipose tissue mass. Proinflammatory cytokines are secreted by adipocytes and macrophages in adipose tissue $[1,20]$. Hypertrophic adipocytes [21] and macrophages shifted the polarization from the M2 to the M1 phenotype [22] frequently secrete $\mathrm{TNF} \alpha$ and other cytokines. In the present study, hypertrophic adipocytes with diameters $>130 \mu \mathrm{m}$ were sel- dom observed in diabetic rats. The relative lack of large hypertrophic adipocytes may partially account for the fact that there was no significant difference in adipose TNF $\alpha$ expression between the diabetic and healthy control rats. However, both adipocyte size and tissue mass were significantly larger in the diabetic than in the healthy control rats. Thus, the diabetic rats could be more strongly affected by proinflammatory cytokines than the healthy control rats.

Previous studies reported that hyperbaric treatment improves hyperglycemia and hyperinsulinemia in both obese [13] and nonobese type 2 diabetic animals [14, 15]. Nevertheless, the mechanism for this improvement remains unknown. In the present study, regular hyperbaric normoxia at 1.3 ATA improved glucose and insulin levels after glucose administration in diabetic rats. No progressive weight loss, hyperglycemia, or hypoinsulinemia [23] was detected in obese type 2 diabetic rats treated with 1.3ATA. The present study revealed that the efficacy of hyperbaric treatment is associated with improvements in insulin sensitivity and glucose tolerance, downregulation of $\mathrm{TNF} \alpha$ expression in adipose tissue, and upregulation of IL-10 in the skeletal muscle. It is widely accepted that IL-10 inhibits proinflammatory cytokine signaling induced by $\mathrm{TNF} \alpha$ and others [24]. IL10 also has efficacy in insulin sensitivity and glucose tolerance in type 2 diabetes with obesity. Kim et al. reported that acute IL-10 treatment improved whole-body insulin action and glucose metabolism during a hyperinsulinemiceuglycemic clamp [25]. Bhargava et al. reported that improvements in insulin sensitivity and glucose tolerance in obese mice were mediated by increased IL-10 production [26]. Grant et al. reported that IL-10 upregulation with concomitant TNF $\alpha$ downregulation improved insulin sensitivity and glucose tolerance in obese mice [27]. IL-10 is expressed and released by the skeletal muscle [28]. It is upregulated during physical exercise [29]. In addition to physical exercise, hyperbaric treatment increases IL-10 expression in injured skeletal muscles [30]. In health, IL10 originating from the skeletal muscle interacts with adipose and other tissues [31]. Therefore, decreased TNF $\alpha$ expression and increased IL-10 expression in diabetic rats under hyperbaric treatment participate in the crosstalk between the adipose tissue and skeletal muscle. Increased tissue oxygenation by hyperbaric treatment could promote the production of anti-inflammatory IL-10 in the skeletal muscles and decrease the production of proinflammatory TNF $\alpha$ in the adipose tissues through inhibition of nuclear factor-kappa B. However, the reasons that IL-10 was increased by hyperbaric treatment were found not in the adipose tissue but in the skeletal muscles; also, as to why IL- 6 and IL- $1 \beta$ were not influenced by the treatment is still unclear in the present study.

Regular hyperbaric treatment improved glucose and insulin levels after glucose administration in diabetic rats. Nevertheless, it had no efficacy for obesity or hyperlipidemia. Moreover, no acute reduction in the glucose level was observed after hyperbaric treatment in the supplementary experiment. Thus, hyperbaric treatment does not promote glucose consumption or inhibit lipid accumulation; rather, it accelerates glucose uptake. 


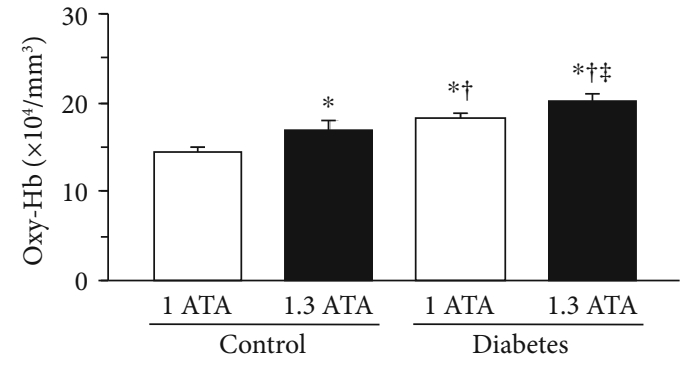

(a)

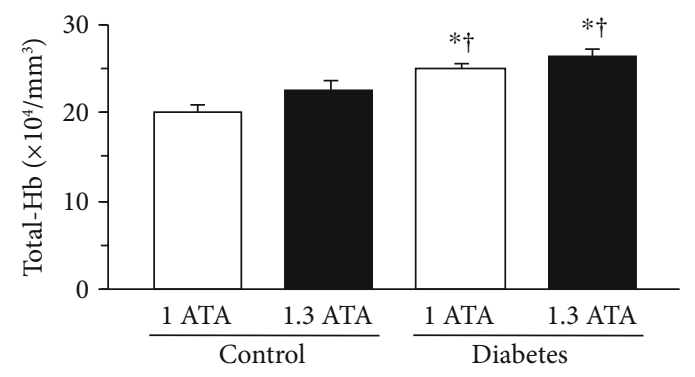

(c)

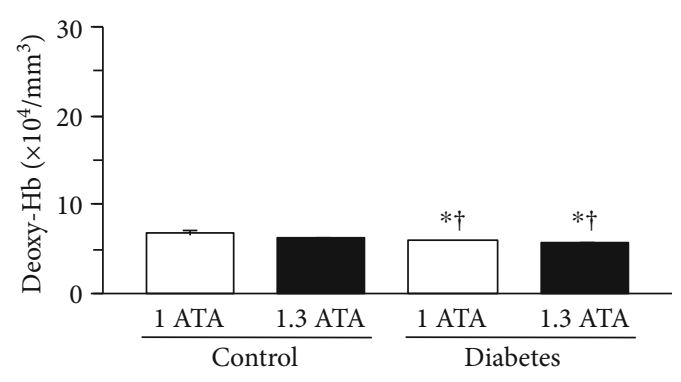

(b)

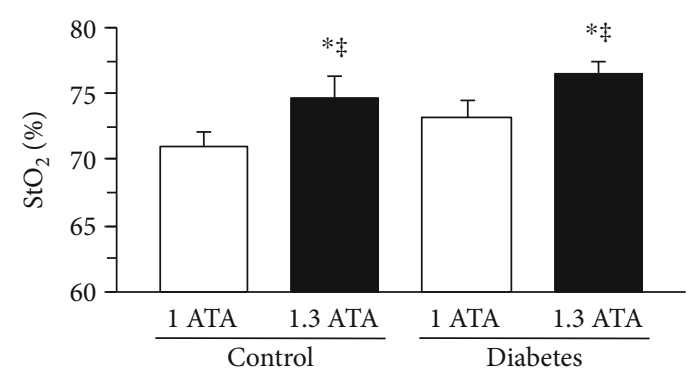

(d)

Figure 6: Skeletal muscle hemodynamic response to hyperbaric treatment. Oxy- $\mathrm{Hb}$ (a), Deoxy-Hb (b), Total- $\mathrm{Hb}$ (c), and StO 2 (d). Values are means \pm standard deviation. ${ }^{*,+}$, Significantly different from the Control, Control HB, and Diabetes groups, $p<0.05$, respectively.

In the present study, skeletal muscle mass and fiber size were significantly smaller in the diabetic than in the healthy control rats. Hyperbaric treatment did not have any influence on the skeletal muscle mass defects in diabetic rats. Skeletal muscle atrophy usually occurs in type 2 diabetes and is controlled by the action of $\mathrm{TNF} \alpha$ on the protein degradation pathway [32]. Conversely, TNF $\alpha$ downregulation in the skeletal muscle inhibits protein degradation [33]. In the present study, there was no significant difference among all groups in terms of the TNF $\alpha$ expression level in both the plantaris and soleus muscles. In a preliminary study, there was no significant difference between the OLETF and LETO rats in terms of the muscle RING finger 1 (E3 ubiquitin ligase) expression level (data not shown). Our previous study [11] showed that the skeletal muscle mass is nearly the same at 24 and 40 wks in OLETF rats ( $200 \mathrm{~g}$ in the soleus muscle). Therefore, structural protein degradation did not occur during this time interval. Skeletal muscle atrophy in type 2 diabetes is associated with physical inactivity, reduced food intake, and peripheral neuropathy. Therefore, the defects in the skeletal muscle mass of OLETF rats may have been caused by the downregulation of protein synthesis which may occur as a result of physical inactivity [34, 35], immobilization [36, 37], denervation [38], and cachexia [39]. Hyperbaric treatment does not seem to enhance structural protein synthesis in the skeletal muscle.

The present study revealed that hyperbaric treatment at 1.3 ATA upregulates IL-10 in the skeletal muscle and improves insulin sensitivity and glucose tolerance in type 2 diabetes with obesity. Nevertheless, this study has certain limitations. The mechanism by which hyperbaric treatment upregulates IL-10 in the skeletal muscle has not been elucidated. It was proposed that obesity causes chronic hypoxia and hypoxia-inducible dysfunction which, in turn, contribute to insulin resistance and glucose intolerance [40]. Therefore, tissue oxygenation during hyperbaric treatment may reverse hypoxia-inducible dysfunction. Hypoxia and hypoxia-related genes increased in the adipose tissue of obese animals. In contrast, no hypoxia-related gene upregulation was detected in the skeletal muscle [41]. In the present study, there was no significant difference between diabetic and healthy control rats in terms of $\mathrm{StO}_{2}$ in their calf muscles. Although hypoxia was not observed in the skeletal muscle, hyperbaric treatment increased oxygenation which, in turn, upregulated IL-10 in this tissue. Therefore, several factors may be involved in IL-10 upregulation in the skeletal muscle. Certain cytokines derived from skeletal muscle fibers may affect adipocytes and improve glucose tolerance [42]. In the present study, however, it was unclear whether IL-10 originating in the skeletal muscle has an endocrine-like impact on TNF $\alpha$ expression in adipose tissue. Further research is required to elucidate the mode of action of hyperbaric treatment in type 2 diabetes with obesity. Additionally, the study to find out the optimum intervention term is also needed to generalize the treatment in the patients.

\section{Conclusion}

Regular hyperbaric treatment with normal air at 1.3 ATA significantly lowered blood glucose and insulin levels, upregulated anti-inflammatory IL-10 in the skeletal muscle, and downregulated proinflammatory $\mathrm{TNF} \alpha$ in adipose tissue of rats with obese type 2 diabetes compared to untreated rats with the same condition. Hyperbaric treatment did not promote glucose consumption, inhibit lipid accumulation, or 
enhance structural protein synthesis in the skeletal muscle. However, hyperbaric treatment would rather accelerate glucose uptake.

\section{Data Availability}

The data that support the findings of this study are available from the corresponding author upon reasonable request.

\section{Disclosure}

Some of the results in the present study have been presented at the American College of Sports Medicine 2019 annual meeting.

\section{Conflicts of Interest}

There are no conflicts of interest to declare.

\section{Authors' Contributions}

NF conceived and designed the experiments. NF, NG, TN, and $\mathrm{WN}$ performed the experiments. NF, NG, TN, WN, and SU analyzed the data. NF, TO, HN, and SU contributed reagents, materials, and analysis tools for the research. NF wrote the manuscript.

\section{Acknowledgments}

This work was carried out at the Analysis Center of Life Science, Natural Science for Basic Research and Development, Hiroshima University. We would like to thank the staff members at that institution for the technical support. This study was supported by a Grant-in-Aid for Scientific Research from the Japanese Ministry of Education, Culture, Sports, Science, and Technology (16K01505, 19K11346).

\section{References}

[1] O. Osborn and J. M. Olefsky, "The cellular and signaling networks linking the immune system and metabolism in disease," Nature Medicine, vol. 18, no. 3, pp. 363-374, 2012.

[2] G. S. Hotamisligil, "Inflammation and metabolic disorders," Nature, vol. 444, no. 7121, pp. 860-867, 2006.

[3] M. Bastien, P. Poirier, I. Lemieux, and J. P. Després, “Overview of epidemiology and contribution of obesity to cardiovascular disease," Progress in Cardiovascular Diseases, vol. 56, no. 4, pp. 369-381, 2014.

[4] I. Tabas and C. K. Glass, "Anti-inflammatory therapy in chronic disease: challenges and opportunities," Science, vol. 339, no. 6116, pp. 166-172, 2013.

[5] H. Yaribeygi, S. L. Atkin, M. Ramezani, and A. Sahebkar, "A review of the molecular pathways mediating the improvement in diabetes mellitus following caloric restriction," Journal of Cellular Physiology, vol. 234, no. 6, pp. 8436-8442, 2019.

[6] H. Yaribeygi, S. L. Atkin, L. E. Simental-Mendía, and A. Sahebkar, "Molecular mechanisms by which aerobic exercise induces insulin sensitivity," Journal of Cellular Physiology, vol. 234, no. 8, pp. 12385-12392, 2019.

[7] C. Popa, M. G. Netea, P. L. C. M. van Riel, J. W. M. van der Meer, and A. F. H. Stalenhoef, "The role of TNF- $\alpha$ in chronic inflammatory conditions, intermediary metabolism, and cardiovascular risk," Journal of Lipid Research, vol. 48, no. 4, pp. 751-762, 2007.

[8] M. F. Neurath and S. Finotto, "IL-6 signaling in autoimmunity, chronic inflammation and inflammation- associated cancer," Cytokine \& Growth Factor Reviews, vol. 22, no. 2, pp. 83-89, 2011.

[9] D. B. Ballak, R. Stienstra, C. J. Tack, C. A. Dinarello, and J. van Diepen, "IL-1 family members in the pathogenesis and treatment of metabolic disease: focus on adipose tissue inflammation and insulin resistance," Cytokine, vol. 75, no. 2, pp. 280-290, 2015.

[10] American College of Sports Medicine, ACSM's Guidelines for Exercise Testing and Prescription, Wolters Kluwer, Philadelphia, PA, 2017.

[11] N. Fujita, S. Aono, K. Karasaki et al., "Changes in lipid metabolism and capillary density of the skeletal muscle following low-intensity exercise training in a rat model of obesity with hyperinsulinemia," PLoS One, vol. 13, no. 5, article e0196895, 2018.

[12] E. M. Camporesi and G. Bosco, "Mechanisms of action of hyperbaric oxygen therapy," Undersea \& Hyperbaric Medicine, vol. 41, no. 3, pp. 247-252, 2014.

[13] N. Fujita, F. Nagatomo, S. Murakami, H. Kondo, A. Ishihara, and H. Fujino, "Effects of hyperbaric oxygen on metabolic capacity of the skeletal muscle in type 2 diabetic rats with obesity," The Scientific World Journal, vol. 2012, Article ID 637978, 9 pages, 2012.

[14] F. Nagatomo, R. R. Roy, H. Takahashi, V. R. Edgerton, and A. Ishihara, "Effect of exposure to hyperbaric oxygen on diabetes-induced cataracts in mice," Journal of Diabetes, vol. 3, no. 4, pp. 301-308, 2011.

[15] K. Yasuda, N. Aoki, T. Adachi et al., "Hyperbaric exposure with high oxygen concentration inhibits growth-associated increase in the glucose level of diabetic Goto-Kakizaki rats," Diabetes Obesity \& Metabolism, vol. 8, no. 6, pp. 714-715, 2006.

[16] K. Punkt, A. Naupert, and G. Asmussen, "Differentiation of rat skeletal muscle fibres during development and ageing," Acta Histochemica, vol. 106, no. 2, pp. 145-154, 2004.

[17] M. Niimi, M. Sato, R. Yokote, S. Tada, and J. Takahara, "Effects of central and peripheral injection of leptin on food intake and on brain Fos expression in the Otsuka Long-Evans Tokushima Fatty rat with hyperleptinaemia," Journal of Neuroendocrinology, vol. 11, no. 8, pp. 605-611, 1999.

[18] J. Ye and O. P. McGuinness, "Inflammation during obesity is not all bad: evidence from animal and human studies," American Journal of Physiology-Endocrinology and Metabolism, vol. 304, no. 5, pp. E466-E477, 2013.

[19] B. Workeneh and M. Bajaj, "The regulation of muscle protein turnover in diabetes," The International Journal of Biochemistry \& Cell Biology, vol. 45, no. 10, pp. 2239-2244, 2013.

[20] A. Majdoubi, O. A. Kishta, and J. Thibodeau, "Role of antigen presentation in the production of pro-inflammatory cytokines in obese adipose tissue," Cytokine, vol. 82, pp. 112-121, 2016.

[21] L. Russo and C. N. Lumeng, "Properties and functions of adipose tissue macrophages in obesity," Immunology, vol. 155, no. 4, pp. 407-417, 2018.

[22] D. Thomas and C. Apovian, "Macrophage functions in lean and obese adipose tissue," Metabolism, vol. 72, pp. 120143, 2017. 
[23] R. S. Rector, J. P. Thyfault, G. M. Uptergrove et al., "Mitochondrial dysfunction precedes insulin resistance and hepatic steatosis and contributes to the natural history of non-alcoholic fatty liver disease in an obese rodent model," Journal of Hepatology, vol. 52, no. 5, pp. 727-736, 2010.

[24] D. F. Fiorentino, M. W. Bond, and T. R. Mosmann, "Two types of mouse T helper cell. IV. Th2 clones secrete a factor that inhibits cytokine production by Th1 clones," Journal of Experimental Medicine, vol. 170, no. 6, pp. 2081-2095, 1989.

[25] H. J. Kim, T. Higashimori, S. Y. Park et al., "Differential effects of interleukin- 6 and -10 on skeletal muscle and liver insulin action in vivo," Diabetes, vol. 53, no. 4, pp. 1060-1067, 2004.

[26] P. Bhargava, C. Li, K. J. Stanya et al., "Immunomodulatory glycan LNFPIII alleviates hepatosteatosis and insulin resistance through direct and indirect control of metabolic pathways," Nature Medicine, vol. 18, no. 11, pp. 1665-1672, 2012.

[27] L. Grant, K. D. Shearer, A. Czopek et al., "Myeloid-cell protein tyrosine phosphatase-1B deficiency in mice protects against high-fat diet and lipopolysaccharide-induced inflammation, hyperinsulinemia, and endotoxemia through an IL-10 STAT3-dependent mechanism," Diabetes, vol. 63, no. 2, pp. 456-470, 2014.

[28] A. M. Petersen and B. K. Pedersen, "The anti-inflammatory effect of exercise," Journal of Applied Physiology, vol. 98, no. 4, pp. 1154-1162, 2005.

[29] J. M. Peake, P. Della Gatta, K. Suzuki, and D. C. Nieman, "Cytokine expression and secretion by skeletal muscle cells: regulatory mechanisms and exercise effects," Exercise Immunology Review, vol. 21, pp. 8-25, 2015.

[30] N. Fujita, M. Ono, T. Tomioka, and M. Deie, "Effects of hyperbaric oxygen at 1.25 atmospheres absolute with normal air on macrophage number and infiltration during rat skeletal muscle regeneration," PLoS One, vol. 9, no. 12, article e115685, 2014.

[31] L. G. Leal, M. A. Lopes, and M. L. Batista Jr., "Physical exercise-induced myokines and muscle-adipose tissue crosstalk: a review of current knowledge and the implications for health and metabolic diseases," Frontiers in Physiology, vol. 9, 2018.

[32] J. Zhou, B. Liu, C. Liang, Y. Li, and Y. H. Song, "Cytokine signaling in skeletal muscle wasting," Trends in Endocrinology and Metabolism, vol. 27, no. 5, pp. 335-347, 2016.

[33] S. Al-Nassan, N. Fujita, H. Kondo, S. Murakami, and H. Fujino, "Chronic exercise training down-regulates TNF- $\alpha$ and atrogin-1/MAFbx in mouse gastrocnemius muscle atrophy induced by hindlimb unloading," Acta Histochemica et Cytochemica, vol. 45, no. 6, pp. 343-349, 2012.

[34] N. Fujita, S. Murakami, T. Arakawa, A. Miki, and H. Fujino, "The combined effect of electrical stimulation and resistance isometric contraction on muscle atrophy in rat tibialis anterior muscle," Bosnian Journal of Basic Medical Sciences, vol. 11, no. 2, pp. 74-79, 2011.

[35] N. Fujita, S. Murakami, and H. Fujino, "The combined effect of electrical stimulation and high-load isometric contraction on protein degradation pathways in muscle atrophy induced by hindlimb unloading," Journal of Biomedicine and Biotechnology, vol. 2011, Article ID 401493, 8 pages, 2011.

[36] N. Fujita, T. Arakawa, T. Matsubara, H. Ando, and A. Miki, "Influence of fixed muscle length and contractile properties on atrophy and subsequent recovery in the rat soleus and plantaris muscles," Archives of Histology and Cytology, vol. 72, no. 3, pp. 151-163, 2009.
[37] N. Fujita, T. Fujimoto, H. Tasaki, T. Arakawa, T. Matsubara, and A. Miki, "Influence of muscle length on muscle atrophy in the mouse tibialis anterior and soleus muscles," Biomedical Research, vol. 30, no. 1, pp. 39-45, 2009.

[38] A. Matsumoto, N. Fujita, T. Arakawa, H. Fujino, and A. Miki, "Influence of electrical stimulation on calpain and ubiquitinproteasome systems in the denervated and unloaded rat tibialis anterior muscles," Acta Histochemica, vol. 116, no. 5, pp. $936-$ 942, 2014.

[39] N. Fujita, H. Fujino, H. Sakamoto, J. Takegaki, and M. Deie, "Time course of ubiquitin-proteasome and macroautophagylysosome pathways in skeletal muscle in rats with heart failure," Biomedical Research, vol. 36, no. 6, pp. 383-392, 2015.

[40] R. Norouzirad, P. Gonzalez-Muniesa, and A. Ghasemi, "Hypoxia in obesity and diabetes: potential therapeutic effects of hyperoxia and nitrate," Oxidative Medicine and Cellular Longevity, vol. 2017, Article ID 5350267, 14 pages, 2017.

[41] J. Ye, Z. Gao, J. Yin, and Q. He, "Hypoxia is a potential risk factor for chronic inflammation and adiponectin reduction in adipose tissue of ob/ob and dietary obese mice," American Journal of Physiology-Endocrinology and Metabolism, vol. 293, no. 4, pp. E1118-E1128, 2007.

[42] B. K. Pedersen, "Anti-inflammatory effects of exercise: role in diabetes and cardiovascular disease," European Journal of Clinical Investigation, vol. 47, no. 8, pp. 600-611, 2017. 


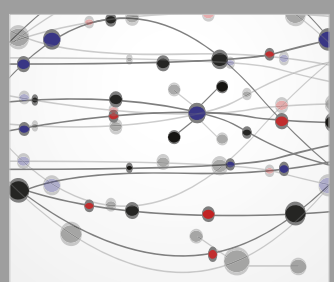

The Scientific World Journal
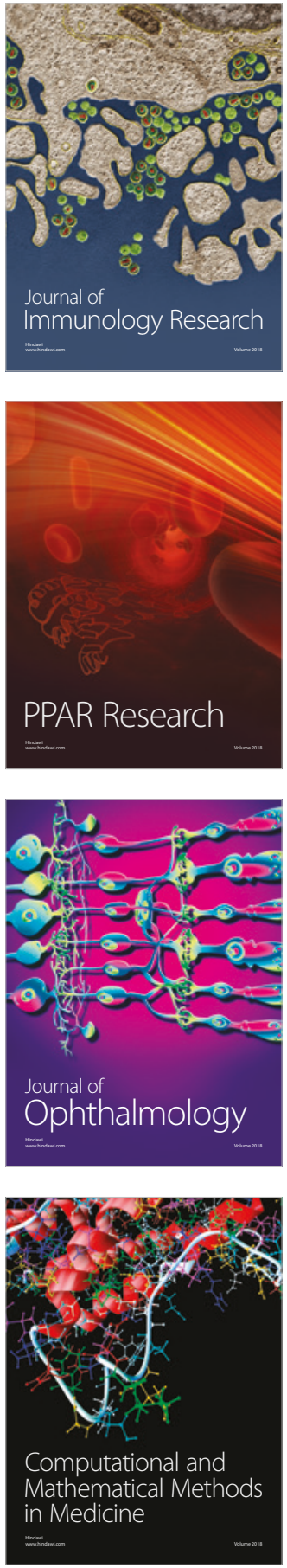

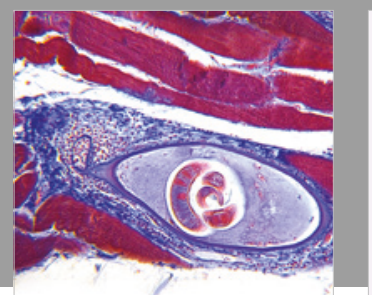

Gastroenterology Research and Practice

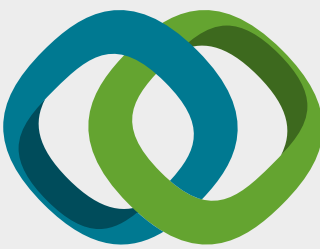

\section{Hindawi}

Submit your manuscripts at

www.hindawi.com
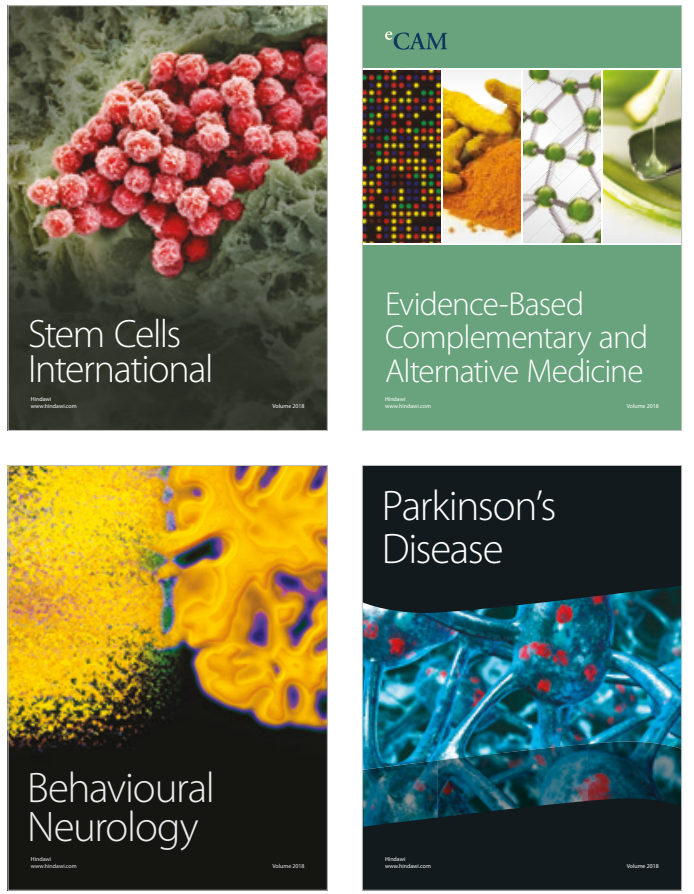

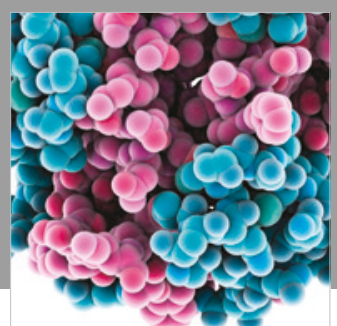

ournal of

Diabetes Research

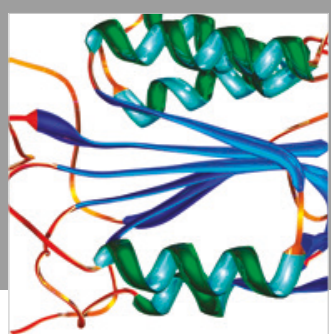

Disease Markers
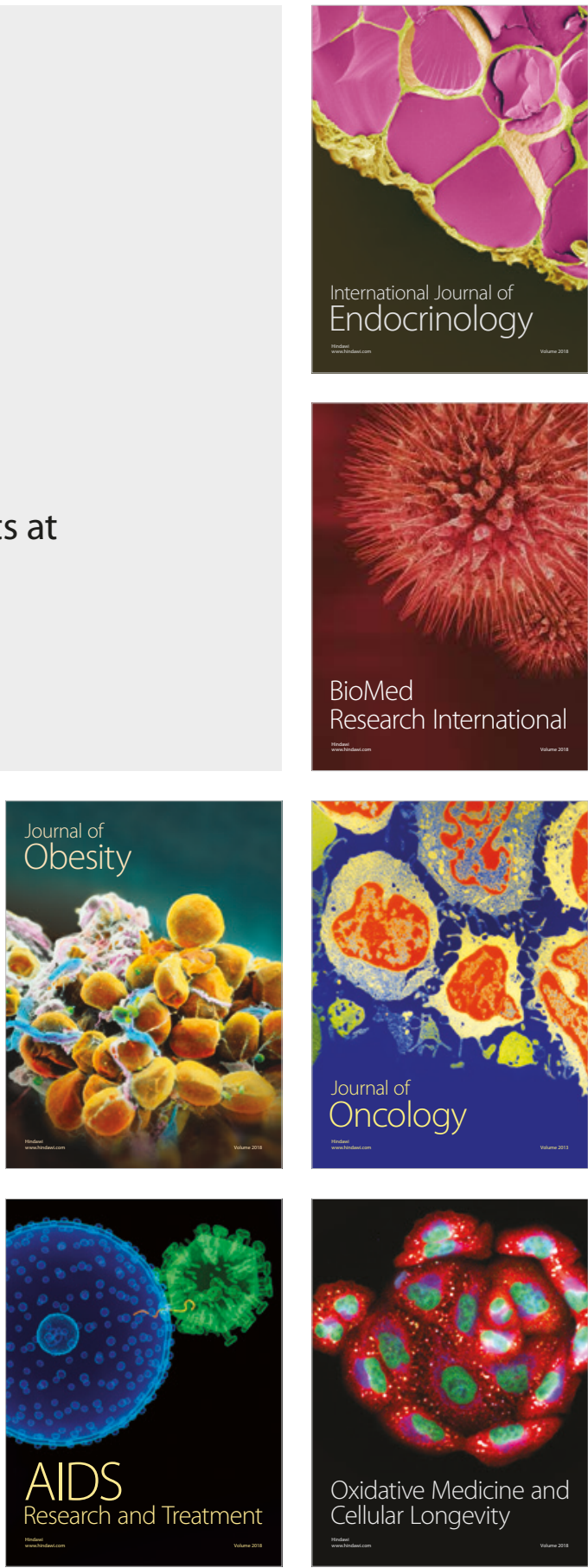